\title{
CONTROLE QUÍMICO DA FERRUGEM (HEMILEIA VASTATRIX BERK \& BR.) DO CAFEEIRO ATRAVÉS DE DIFERENTES ESQUEMAS DE APLICAÇÃO'1
}

\author{
SÁRA MARIA CHALFOUN² e VICENTE LUIZ DE CARVALHO ${ }^{3}$
}

\begin{abstract}
RESUMO - O presente trabalho foi desenvolvido com o objetivo de testar os diferentes esquemas de controle químico da ferrugem do cafeeiro disponíveis, durante o período de 1994 a 1996 em São Sebastião do Paraíso, MG. Os tratamentos foram constituídos por esquemas preventivos, baseados em fungicidas cúpricos (oxicloreto de cobre e óxido cuproso), curativos, baseados em fungicidas sistêmicos (grupo dos triazois) e mistos (associação de esquemas curativos e preventivos). As datas de aplicação foram previamente fixadas. Os resultados obtidos demonstraram que: a) a aplicação de esquemas de controle por meio de datas pré-fixadas tem permitido a elevação da ferrugem ao final do seu ciclo; b) o fungicida triadimenol 1,5\%, associado ao inseticida dissulfoton $7,5 \%$, granulado, aplicado ao solo no mês de dezembro, na dosagem de $40 \mathrm{~kg} /$ ha do produto comercial, foi o único tratamento que manteve baixos índices de ferrugem durante o ciclo da doença; c) os tratamentos aplicados por meio do sistema radicular, triadimenol e cyproconazole, foram beneficiados pela associação com o inseticida dissulfoton.

Termos para indexação: Coffea arabica, eficácia de controle, ferrugem, fungicidas, épocas de aplicação.
\end{abstract}

\section{CHEMICAL CONTROL OF COFFEE RUST (HEMILEIA VASTATRIX BERK \& BR.) THROUGH DIFFERENT APPLICATION SCHEMES}

\begin{abstract}
The present work was developed to test different available coffee rust chemical control schemes during the period of 1994 to 1996, at São Sebastião do Paraíso, MG, Brazil. The treatments were performed by preventive schemes, based on copper fungides (copper oxycloride and copper oxide) curative ones based in systemic fungicides (triazol chemical group) and mixed ones (association of curatives and preventive schemes). The application periods were previously fixed. The result obtained showed that: a) the coffee rust control measures applied at previously periods have permited the increase of disease levels at the end of its cycle; b) the systemic fungicide triadimenol 1,5\%, associated with the insecticide disulfoton 7,5\%, granulate formulation, applied to the soil in December at $40 \mathrm{~kg} / \mathrm{ha}$ of the commercial product was the only treatment that has maintened low levels of coffee rust during the disease cycle, and c) the effect of treatments applied to the soil, triadimenol and cyproconazole, was improved by the association with the insecticide disulfoton, enhancing the coffee rust control.
\end{abstract}

Index terms: Coffea arabica, control efficacy, rust, fungicides, application periods.

\section{INTRODUÇÃO}

Os fungicidas à base de cobre foram, por muito tempo, quase que os únicos produtos utilizados no controle de ferrugem-do-cafeeiro, até que, a partir

\footnotetext{
${ }^{1}$ Aceito para publicação em 29 de junho de 1998.

${ }^{2}$ Eng. Agr., Dr., EPAMIG, Caixa Postal 176, CEP 37200-000 Lavras, MG. E-mail: chalfoun@ufla.br

${ }^{3}$ Eng. Agr., M.Sc., EPAMIG.
}

dos anos 60, surgiram novos produtos de diferentes composições químicas, alguns de amplo espectro, e outros, de uso mais específico (Becker-Raterink et al., 1991).

Embora os fungicidas cúpricos (protetores) apresentem eficiência comprovada no controle a ferrugem, desde que aplicados a intervalos de semanas (Bock, 1962), os fungicidas sistêmicos apresentam vantagens em relação aos protetores por exercerem efeito curativo sobre as lesões novas e a inibição de 
esporulação das lesões velhas (Nunes, 1986). Os fungicidas sistêmicos, entre eles os do grupo químico dos triazóis, lançados no mercado após 1976, têm demonstrado elevada eficiência na redução do inóculo residual, permitindo o retardamento do início das pulverizações e a redução de seu número, quando aplicados por meio de pulverizações ou via sistema radicular (Silva-Acunã et al., 1993).

A formulação dos fungicidas sistêmicos na forma granulada, associados ou não a um inseticida possibilitando sua aplicação ao solo, proporcionou uma alternativa válida para o controle da ferrugem em áreas de topografia acidentada, sem disponibilidade de água (Silva-Acunã et al., 1993), em plantios adensados e em lavouras extensivas. Almeida \& Matiello (1989) e Bordin et al. (1989), citados por Souza (1991), verificaram que, no caso da formulação granulada do produto triadimenol associado com o inseticida dissulfoton, houve efeito sinérgico positivo do inseticida, reduzindo a infecção e melhorando a ação do triadimenol nas doses baixas e médias.

Deve-se observar, no entanto, que o sucesso da aplicação de medidas de controle químico depende, ainda, do preenchimento de exigências inerentes aos fungicidas utilizados, como no caso dos aplicados via sistema radicular, que exigem a presença de umidade e um período de absorção e translocação para concentração do produto nas folhas a um nível tal que o mesmo atue eficientemente sobre o fungo (Nunes, 1986; Deall, 1990).

Para garantir o êxito do controle químico, é ainda indispensável a aplicação do fungicida no momento apropriado, de acordo com o ciclo da doença e sua dinâmica no transcurso do ano. Esta, por sua vez, encontra-se relacionada com as variações climáticas ocorridas durante o ano, uma vez que elas afetam o patógeno, o hospedeiro, e, conseqüentemente, as relações patógeno e hospedeiro (Moraes, 1983; Chalfoun \& Lima, 1986).

Sabe-se, também, que a alta produção, que promove um desequilíbrio nutricional, torna os cafeeiros mais predispostos à ocorrência da ferrugem, tendo-se observado uma correlação entre a produção e a incidência de ferrugem que é sempre maior nos anos de maior produção (Carvalho et al., 1996).

Com relação ao limite econômico para o controle da doença, é calculado em torno de $10 \%$ de infecção no ponto máximo da epidemia, que é um nível de controle sob o qual provavelmente não seria possível obter um aumento da produção com aplicações adicionais (Waller, 1982).

O presente trabalho teve como objetivo testar diferentes esquemas de controle da ferrugem-do-cafeeiro envolvendo diferentes fungicidas (preventivos, mistos e curativos) aplicados em épocas pré-fixadas.

\section{MATERIAL E MÉTODOS}

O experimento foi instalado na Fazenda Experimental da EPAMIG, no município de São Sebastião do Paraíso, durante o ano agrícola 1994/95.

A lavoura utilizada foi constituída pela cultivar Mundo Novo 379/19, plantada em espaçamento 3,5 x 2,0 m.

O delineamento experimental utilizado foi o de blocos ao acaso, com quatro repetições dos tratamentos a seguir, nos quais as dosagens dos defensivos referem-se à dosagem dos produtos comerciais: 1) fungicida cúprico, oxicloreto de cobre, $50 \%$ de cobre metálico, pó molhável, na dosagem de $4 \mathrm{~kg} / \mathrm{ha}$ do produto comercial, aplicado no período de dezembro de 1995 a março de 1996 (4 aplicações); 2) fungicida sistêmico triadimenol, $25 \%$ de ingrediente ativo, na dosagem de $1 \mathrm{~L} / \mathrm{ha}$, concentrado emulsionável, aplicado em dezembro, e fungicida cúprico, óxido cuproso, $80 \%$ de cobre metálico, pó molhável, na dosagem de $3 \mathrm{~kg} / \mathrm{ha}$, aplicado em janeiro e fevereiro; 3) fungicida sistêmico triadimenol $25 \%$ de ingrediente ativo, na dosagem de 1,0 L/ha, concentrado emulsionável, aplicado em janeiro e fevereiro; 4) fungicida sistêmico triadimenol $6 \%$ de ingrediente ativo, granulado, na dosagem de $20 \mathrm{~kg} / \mathrm{ha}$, aplicado em dezembro; 5) fungicida sistêmico triadimenol $1,5 \%$ + dissulfoton $7,5 \%$, granulado, na dosagem de $50 \mathrm{~kg} / \mathrm{ha}$, aplicado em dezembro; 6) fungicida cúprico, óxido cuproso, $80 \%$ de cobre metálico, na dosagem de $3 \mathrm{~kg} / \mathrm{ha}$, aplicado em dezembro, e fungicida sistêmico, cyproconazole, $10 \%$ de ingrediente ativo, na dosagem de $0,5 \mathrm{~L} / \mathrm{ha}$, aplicado em janeiro e março; 7) fungicida sistêmico, cyproconazole $0,4 \%$ + dissulfoton $10 \%$, granulado, na dosagem de $40 \mathrm{~kg} / \mathrm{ha}$, aplicado em outubro e fungicida cúprico, óxido cuproso, $80 \%$ de cobre metálico, na dosagem de $3 \mathrm{~kg} / \mathrm{ha}$, aplicado em dezembro; 8) fungicida cúprico, oxicloreto de cobre, $50 \%$ de cobre metálico, na dosagem de $4 \mathrm{~kg} / \mathrm{ha}$, aplicado em dezembro, fungicida cúprico oxicloreto de cobre $50 \%$, na dosagem de $4 \mathrm{~kg} / \mathrm{ha}+$ fungicida sistêmico cyproconazole $20 \%$, na dosagem de $0,3 \mathrm{~L} / \mathrm{ha}$ (mistura de tanque), aplicado em janeiro, e fungicida cúprico, oxicloreto de cobre, $50 \%$ de cobre metálico, na dosagem de $3 \mathrm{~kg} / \mathrm{ha}$, aplicado em março; 9) fungicida sistêmico, cyproconazole $10 \%$, 
granulado, na dosagem de $20 \mathrm{~kg} / \mathrm{ha}$, aplicado em outubro, e fungicida cúprico, óxido cuproso, $80 \%$ de cobre metálico, na dosagem de $3 \mathrm{~kg} / \mathrm{ha}$, aplicado em dezembro; 10) testemunha não tratada.

As parcelas foram constituídas por 28 plantas, sendo a área útil formada por dez plantas.

Os fungicidas em formulação pó molhável e concentrado emulsionável foram aplicados por meio de atomizadores costais motorizados, com um gasto médio de 400 litros da calda fungicida por hectare. Os fungicidas granulados foram aplicados em sulcos efetuados na projeção da copa dos cafeeiros.

Durante a condução do experimento foram efetuadas coletas mensais de 100 folhas por parcela, localizadas no terço médio das plantas, e ao redor das mesmas ( $3^{\circ}$ ou $4^{\circ}$ par de folhas), nas quais avaliou-se a freqüência de incidência da ferrugem. Por ocasião da colheita, foram avaliadas as produções correspondentes aos anos de 1995 e 1996, considerando-se que os efeitos negativos da ferrugem incidem principalmente sobre a produção do ano seguinte.

Os resultados obtidos foram analisados pelo teste de Tukey a $5 \%$ de probabilidade.

\section{RESULTADOS E DISCUSSÃO}

Observou-se que, de maneira geral, o desenvolvimento mais tardio da ferrugem na área experimental em relação à época de aplicação dos tratamentos prejudicou a eficiência dos diferentes esquemas de controle, o que permitiu uma elevação da doença ao final do ciclo da mesma.

Os resultados obtidos (Tabela 1) indicam que até o mês de fevereiro de 1995 os índices de ferrugem mantiveram-se baixos, inclusive nas plantas não pulverizadas (tratamento 10). Por outro lado, foram observados índices elevados de ferrugem, ao final do ciclo da doença, como pode ser observado nos tratamentos 1, 2 e 3, e em junho de 1995 tais índices atingiram valores superiores a $50 \%$ de folhas infectadas, valores estes semelhantes aos índices atingidos nas plantas não pulverizadas, e acima de $20 \%$ nos demais tratamentos, exceto no tratamento 5, no qual o índice de ferrugem manteve-se baixo até o final do ciclo da doença.

Desta forma, a aplicação de fungicida cúprico via foliar exclusivamente preventiva mostrou-se ineficiente no controle da ferrugem, quando aplica- do mensalmente no período de dezembro de 1995 a março de 1996 (tratamento 1), uma vez que, aplicado antecipadamente e com um poder residual de 3 a 4 semanas (Bock, 1962), não estava mais presente na planta por ocasião do período de maior desenvolvimento da doença, a qual ocorreu a partir do mês de março de 1996.

Os tratamentos com produtos sistêmicos (tratamentos 2 e 3), associados ou não a fungicidas cúpricos, apesar do maior poder residual dos fungicidas sistêmicos aplicados às folhas, e apesar, também, do efeito curativo desses fungicidas, quando comparados aos fungicidas preventivos, sua aplicação antecipada na área experimental, com baixos índices da doença por ocasião da aplicação dos fungicidas permitiu a elevação desses índices ao final do ciclo (junho de 1996). O ideal, nesta modalidade de controle, seria utilizar as propriedades curativas e outras propriedades destes produtos, para retardar o início das pulverizações e aumentar o intervalo entre elas.

Os fungicidas sistêmicos foliares, associados a fungicidas cúpricos (tratamentos 6 e 8), apresentaram um resultado intermediário aos anteriores em relação ao controle da ferrugem, graças à melhor utilização das propriedades dos fungicidas sistêmicos citadas anteriormente quanto ao poder residual dos produtos, o que permitiu um maior prolongamento do período de tratamento (até o mês de março).

Os tratamentos aplicados via sistema radicular (tratamentos 5 e 7), constituídos pelos fungicidas triadimenol e cyproconazole associados ao inseticida dissulfoton, mantiveram índice de ferrugem mais reduzido durante o ciclo da doença, em comparação com os mesmos fungicidas aplicados isoladamente (tratamentos 4 e 9), o que confirma a ação sinérgica da associação dos fungicidas citados com o inseticida dissulfoton, melhorando o controle da ferrugem, de acordo com as afirmativas de Almeida \& Matiello (1989) e Bordin et al. (1989), citados por Souza (1991).

Observou-se, em face dos resultados representados na Tabela 2, que no período de novembro de 1994 a março de 1995, a precipitação total excedeu 265,3 mm ao total do mesmo período em 1995/96. Tal fato pode explicar, em parte, o retardamento na evolução da ferrugem, conforme demonstrado em trabalhos anteriores (Moraes, 1983; Chalfoun \& Lima, 1986). 
Considerando-se que o efeito do ataque da ferrugem evidencia-se sobre a produção do ano seguinte, observa-se, no ano agrícola 1995/96, uma tendência de os tratamentos que apresentaram maior redu- ção na incidência de ferrugem, também apresentarem as produções mais elevadas, sendo que as maiores produções registradas foram as dos tratamentos 5 e 7 .

TABELA 1. Controle da ferrugem-do-cafeeiro, e produção em diferentes modalidades de tratamentos. Ano agrícola 1994/95. São Sebastião do Paraíso, MG¹.

\begin{tabular}{|c|c|c|c|c|c|c|c|}
\hline \multirow[t]{3}{*}{ Tratamento } & \multicolumn{5}{|c|}{ Folhas infectadas $(\%)$} & \multirow{2}{*}{\multicolumn{2}{|c|}{$\begin{array}{c}\text { Produção } \\
\text { (sc } 60 \text { kg/ha) }\end{array}$}} \\
\hline & \multicolumn{5}{|c|}{1995} & & \\
\hline & Fev. & Mar. & Abr. & Maio & Jun. & 1995 & 1996 \\
\hline Oxicloreto de cobre $(50 \%)$ & $2,9 \mathrm{a}$ & $5,4 \mathrm{bcd}$ & 9,3 bcd & $20,5 \mathrm{bc}$ & $51,1 \mathrm{abc}$ & $3,5 \mathrm{a}$ & $20,9 \mathrm{~cd}$ \\
\hline Triadimenol-CE + óxido cuproso $(80 \%)$ & $0,0 \mathrm{a}$ & $2,3 \mathrm{~d}$ & $7,0 \mathrm{bcd}$ & $54,5 \mathrm{a}$ & $75,4 \mathrm{a}$ & $5,3 \mathrm{a}$ & $15,5 \mathrm{~cd}$ \\
\hline Triadimenol (25\%) & $1,1 \mathrm{a}$ & $0,9 \mathrm{~d}$ & $1,7 \mathrm{~d}$ & $23,7 \mathrm{~b}$ & $55,9 \mathrm{ab}$ & $5,5 \mathrm{a}$ & $15,2 \mathrm{~cd}$ \\
\hline Triadimenol - GR & $3,7 \mathrm{a}$ & $12,1 \mathrm{ab}$ & $13,0 \mathrm{ab}$ & $21,1 \mathrm{bc}$ & 30,1 cde & $6,7 \mathrm{a}$ & $22,4 \mathrm{bc}$ \\
\hline Triadimenol $(1,5 \%)+$ dissulfoton $(7,5 \%)$ & $3,9 \mathrm{a}$ & $9,6 \mathrm{abc}$ & 6,9 bcd & $8,9 \mathrm{bc}$ & $12,1 \mathrm{e}$ & 4,8 a & $37,2 \mathrm{ab}$ \\
\hline Óxido cuproso $(80 \%)+$ cyproconazole $(10 \%)$ & $0,5 \mathrm{a}$ & $0,7 \mathrm{~d}$ & $1,5 \mathrm{~d}$ & $6,1 \mathrm{bc}$ & 39,5 bcd & $2,3 \mathrm{a}$ & $23,7 \mathrm{bc}$ \\
\hline Cyproconazole $(0,4 \%)+$ dissulfoton $(10 \%)+$ óxido & & & & & & & \\
\hline cuproso $(80 \%)$ & $1,4 \mathrm{a}$ & $2,5 \mathrm{~cd}$ & $2,7 \mathrm{~cd}$ & $11,7 \mathrm{bc}$ & $20,7 \mathrm{de}$ & $3,7 \mathrm{a}$ & 40,8 a \\
\hline Oxicloreto de cobre $(50 \%)+$ cyproconazole $(20 \%)$ & $1,3 \mathrm{a}$ & $1,5 \mathrm{~d}$ & $5,6 \mathrm{bcd}$ & $4,7 \mathrm{c}$ & 28,0 cde & $4,4 \mathrm{a}$ & $25,0 \mathrm{bc}$ \\
\hline Cyproconazole $(10 \%)+$ óxido cuproso $(80 \%)$ & $1,9 \mathrm{a}$ & $5,7 \mathrm{bcd}$ & $12,3 \mathrm{bc}$ & $25,5 \mathrm{~b}$ & $33,9 \mathrm{bcd}$ & $3,5 \mathrm{a}$ & $23,9 \mathrm{bc}$ \\
\hline Testemunha não pulverizada & - & $16,3 \mathrm{a}$ & $26,6 \mathrm{a}$ & $60,6 \mathrm{a}$ & $58,1 \mathrm{ab}$ & $4,0 \mathrm{a}$ & $9,2 \mathrm{~d}$ \\
\hline CV (\%) & 81,7 & 25,1 & 28,9 & 23,6 & 16,0 & 47,6 & 15,1 \\
\hline
\end{tabular}

TABELA 2. Regime de chuvas e temperaturas durante o período 1994 a 1996. São Sebastião do Paraíso, MGํ․

\begin{tabular}{|c|c|c|c|c|c|c|}
\hline \multirow[t]{2}{*}{ Meses } & \multicolumn{2}{|c|}{1994} & \multicolumn{2}{|c|}{1995} & \multicolumn{2}{|c|}{1996} \\
\hline & $\begin{array}{l}\text { Precipitação } \\
(\mathrm{mm})\end{array}$ & $\begin{array}{c}\text { Temperatura } \\
\left({ }^{\circ} \mathrm{C}\right)\end{array}$ & $\begin{array}{l}\text { Precipitação } \\
(\mathrm{mm})\end{array}$ & $\begin{array}{c}\text { Temperatura } \\
\left({ }^{\circ} \mathrm{C}\right)\end{array}$ & $\begin{array}{l}\text { Precipitação } \\
(\mathrm{mm})\end{array}$ & $\begin{array}{c}\text { Temperatura } \\
\left({ }^{\circ} \mathrm{C}\right)\end{array}$ \\
\hline Jan. & 291,8 & 21,8 & 313,0 & 24,3 & 335,1 & 23,8 \\
\hline Fev. & 30,9 & 24,8 & 477,0 & 24,1 & 130,2 & 24,5 \\
\hline Mar. & 151,9 & 22,8 & 145,1 & 23,6 & 180,3 & 24,5 \\
\hline Abr. & 38,4 & 21,9 & 122,2 & 21,5 & 58,8 & 21,8 \\
\hline Maio & 104,8 & 20,3 & 79,0 & 19,5 & 70,4 & 18,7 \\
\hline Jun. & 7,9 & 21,9 & 9,8 & 18,0 & 31,2 & 17,2 \\
\hline Jul. & 12,9 & 23,7 & 12,9 & 19,4 & 0,0 & 17,6 \\
\hline Ago. & 0,0 & 19,9 & 0,0 & 21,5 & 17,6 & 20,0 \\
\hline Set. & 0,0 & 23,2 & 39,8 & 22,3 & 131,0 & 20,8 \\
\hline Out. & 141,4 & 25,1 & 156,6 & 22,1 & 157,6 & 22,5 \\
\hline Nov. & 242,4 & 25,8 & 141,8 & 22,4 & 361,1 & 22,2 \\
\hline Dez. & 309,3 & 23,8 & 434,1 & 23,2 & & \\
\hline
\end{tabular}

${ }^{1}$ Dados obtidos na Estação Meteorológica da Fazenda Experimental da EPAMIG em São Sebastião do Paraíso, MG. 


\section{CONCLUSÕES}

1. A aplicação de esquemas de controle da ferrugem com base em datas pré-fixadas permite uma elevação da doença ao final do seu ciclo.

2. O tratamento com o produto triadimenol $1,5 \%$ associado ao inseticida dissulfoton $7,5 \%$, granulado, aplicado ao solo em dezembro, na dosagem de $40 \mathrm{~kg} / \mathrm{ha}$ do produto comercial, permite a manutenção de baixos índices de ferrugem durante o ciclo da doença.

3. Os tratamentos com triadimenol e cyproconazole, aplicados através do sistema radicular, são beneficiados pela associação com o inseticida dissulfoton, melhorando o controle da ferrugem.

\section{REFERÊNCIAS}

BECKER-RATERINK, S.; MORAES, W.B.C.; QUIJANO-RICO, M. La roya del cafetoconocimiento y control. Deutsche: Gesellschaft für Technishe Zusammenarbeit (GTZ) Gmbh, 1991. $281 \mathrm{p}$.

BOCK, K.R. Control of coffee leaf rust in Kenya Colony. Transactions of British Mycology Society, v.45, n.3, p.301-313, 1962

CARVALHO,V.L. de; CHALFOUN, S.M.; CASTRO, H.A. de; CARVALHO, V.D. de. Influência da produção na incidência da ferrugem do cafeeiro. Pesquisa
Agropecuária Brasileira, Brasília, v.31, n.6, p.401405, jun. 1996.

CHALFOUN, S.M.; LIMA, R.D. de. Influência do clima sobre a incidência de doenças infecciosas. Informe Agropecuário, Belo Horizonte, v.12, n.138, p.3136, jun. 1986.

DEALL, M.W. Studies on the control of coffee rust (Hemileia vastatrix) with Bayfidan GR in Zimbabwe. Pflanzenschutz Nachrichten, v.43, n.3, p.203-216, 1990.

MORAES, S.A. de. A ferrugem do cafeeiro: importância, condições predisponentes, evolução e situação no Brasil. Campinas: Instituto Agronômico, 1983. 50p.

NUNES, A.M.L. Tempo de absorção, efeito protetor, curativo e de translocação de fungicidas no controle da ferrugem do cafeeiro (Hemileia vastatrix Berk \& Br). Viçosa: UFV, 1986. 91p. Tese de Mestrado.

SILVA-ACUNÃ, R.; ZAMBOLIM, L.; GONZALEZMOLINA, E. de C. Controle da ferrugem do cafeeiro com triadimenol via solo, na Venezuela. Fitopatologia Brasileira, v.18, n.1, p.70-75, mar. 1993.

SOUZA, M.T. de. Ação protetora e curativa dos compostos triadimenol e aldicarbe em mudas de cafeeiro no controle da ferrugem causada por Hemileia vastatrix. Lavras: UFLA, 1991. 76p. Tese de Mestrado.

WALLER, J.M. Coffee rust epidemiology and control. Crop Protection, v.1, p.385-404, 1982. 